\title{
La educacionalización del mundo: una mirada genealógica de la modernidad ${ }^{1}$
}

\author{
A educacionalização do mundo: \\ um olhar genealógico da modernidade \\ Educationalization of the world: \\ a genealogical perspective of modernity \\ Carlos Ernesto Noguera-Ramírez \\ Universidad Pedagógica Nacional (Colombia) \\ orcid.org/0000-0001-9848-0724 \\ cnoguera@pedagogica.edu.co \\ Dora Lilia Marín-Díaz \\ Universidad Distrital Francisco José de Caldas (Colombia) \\ orcid.org/0000-0002-0466-3368 \\ dlmarind@udistrital.edu.co
}

\section{Resumen}

Una historia de la educación en clave genealógica es una genealogía de las prácticas pedagógicas. Ahora bien, una genealogía de las prácticas pedagógicas modernas es, finalmente, una historia de la modernidad en clave genealógica. Sobre la base de estas dos tesis y retomando un conjunto de investigaciones realizadas por el Grupo de Historia de la Práctica Pedagógica (GHPP) de Colombia, el artículo se propone discutir el concepto de "educacionalización del mundo" propuesto Tröhler $(2014,2015)$ para sustentar que el acontecimiento llamado modernidad es, fundamentalmente, la educacionalización del mundo. Los conceptos de procedencia y emergencia son las herramientas metodológicas usadas para describir tanto la procedencia pastoral de las prácticas pedagógicas modernas (Foucault, 2007; Hunter, 1998; Sloterdijk, 2012), como la emergencia de un novedoso conjunto de antropotécnicas disciplinarias y con ellas de la escuela como el artefacto sobre el cual se sustenta el proceso de educacionalización moderno y contemporáneo.

Palabras clave: Genealogía. Prácticas pedagógicas. Educacionalización. Modernidad.

\footnotetext{
${ }^{1}$ Este artículo forma parte de los avances de la investigación "Sobre la educación contemporánea: pedagogía, filosofía y antropología", financiada por el Centro de investigaciones - CIUP de la Universidad Pedagógica Nacional (DSI-463-18), Bogotá, Colombia. Partes de este texto fueron presentadas como ponencias en el XIII Congreso Iberoamericano de Historia de la Educación Latinoamericana, Montevideo, 2018.
} 


\begin{abstract}
The history of education in genealogical perspective is a genealogy of pedagogical practices. However, a genealogy of modern pedagogical practices is, finally, a history of modernity in a genealogical key. On the basis of these two theses and retaking a set of investigations carried out by the Group of History of the Pedagogical Practice (GHPP) from Colombia, the article proposes to discuss the concept of "educationalization of the World" proposed by Tröhler $(2014,2015)$ to support that the event called modernity is fundamentally the educationalization of the world. The concepts of provenance and emergence are the methodological tools used to describe both the pastoral origin of modern pedagogical practices (Foucault, 2007; Hunter, 1998; Sloterdijk, 2012), as the emergence of a new set of disciplinary antropotechniques and with them of the school as the artifact on which the process of modern and contemporary education is supported.
\end{abstract}

Key words: Genealogy. Pedagogical practices. Educationalization. Modernity.

\title{
Resumo
}

Uma história da educação em chave genealógica é uma genealogia das práticas pedagógicas. Agora bem, uma genealogia das práticas pedagógicas modernas é, finalmente, uma história da modernidade em chave genealógica. Levando em conta essas duas teses e retomando um conjunto de pesquisas realizadas pelo "Grupo de Historia de la Práctica Pedagógica" (GHPP) da Colômbia, o artigo tem como propósito discutir o conceito de "educacionalização do mundo" proposto por Tröhler $(2014,2015)$ para sustentar que o acontecimento chamado de modernidade é, fundamentalmente, a educacionalização do mundo. Os conceitos de procedência e emergência são as ferramentas metodológicas usadas para descrever tanto a procedência pastoral das práticas pedagógicas modernas (Foucault, 2007; Hunter, 1998; Sloterdijk, 2012), quanto a emergência de um novo conjunto de antropotécnicas disciplinares e com elas da escola como artefato no qual se sustenta o processo de educacionalização moderno e contemporâneo.

Palavras-chave: Genealogia. Práticas pedagógicas. Educacionalização. Modernidade. 
For history researchers, it is not a needless luxury to consider from time to time the content and the significance of the basic concepts they use, certainly if they have the ambition to interpret and/or explain history in addition to purely describing it.

Smeyers y Depaepe (2008, p. 13)

\section{Introducción}

Hace apenas una década, varios filósofos, historiadores y especialistas de la educación europeos publicaron un volumen dedicado al análisis de una problemática que, si bien tenía que ver directamente con el campo de los estudios educacionales, se extendía hacia otros ámbitos de las ciencias sociales y humanas. Se trataba de un asunto de estudio novedoso para el cual tuvieron que acuñar una doble expresión: pedagogización o educacionalización ${ }^{2}$ (Smeyers y Depaepe, 2008). Según Tröhler, uno de los más destacados estudiosos de este asunto,

La palabra educacionalización, concepto nuevo en el actual debate internacional de los estudios culturales y educativos, se refiere a un reflejo cultural que se sitúa en el centro de unos fenómenos que, en su conjunto, se suelen llamar "la modernidad". Es un reflejo cultural que traduce ciertos problemas sociales — que en sí mismos no son educativos - en un problema educativo, y con ello los asigna a la educación (2014, p. 9).

Dos ejemplos significativos de este "reflejo educativo" fueron las reacciones que provocó, en Estados Unidos, el lanzamiento del primer satélite al espacio por parte de la Unión Soviética en 1957 y, más recientemente, el informe A Nation at Risk (1983), encargado por el presidente Ronald Reagan a una comisión nacional. En ambos casos, los análisis y discusiones terminaron en la implementación de importantes reformas educativas, pues parecía evidente que tanto el fracaso tecnológico de 1957 como la crisis económica de la década de 1970 tenían su explicación en el atraso del sistema educativo nacional (Tröhler, 2014).

Desde luego, el fenómeno no se reduce a los Estados Unidos, pues fácilmente encontraríamos en todos los continentes ejemplos de este reflejo, y no se trata de un asunto propio del siglo XX. Según los estudios de Tröhler (2013, 2014), su surgimiento se puede localizar hacia 1800 como resultado de un particular cambio cultural cuya principal figura fue el pedagogo suizo J. H. Pestalozzi (1746-1827). La característica central de ese cambio cultural, producido en Europa del Norte, Europa Occidental y los Estados Unidos de América entre finales del siglo XVIII e inicios del siglo XIX, fue que diversos problemas sociales pasaron a interpretarse como problemas educativos, motivo por el cual se puede hablar de un verdadero "giro educativo" (Tröhler, 2014). En síntesis, el fenómeno señalado con la expresión educacionalización de los problemas sociales, forma parte de un "giro" que

\footnotetext{
${ }^{2}$ Teniendo en cuenta la procedencia lingüística de estos investigadores se utiliza la expresión Educationalization o su correspondiente alemana Pädagogisierung; esta última se traduce al inglés como Pedagogization.
} 
se estableció en el discurso educativo hacia finales del siglo XVIII y posteriormente condujo a la fundación de la escuela moderna en el contexto de las naciones-Estado del siglo XIX. Hoy, el fenómeno sigue vivo y encuentra su expresión en el marco del Banco Mundial, las Naciones Unidas, la UNESCO y la OCDE. Parte de la premisa de que los problemas fundamentales del presente y la planificación del futuro, de hecho, son fundamentalmente cuestiones educativas (Tröhler, 2014, p. 14).

La problemática analizada por Tröhler no es un asunto particular del campo de la historia de la educación, aunque su aporte en este sentido, como veremos, es bastante significativo: más allá de la historia y la filosofía de la educación, constituye una herramienta clave para la comprensión del proceso de constitución de las "sociedades" modernas, pues como señala Jeissman (1995, citado por Tröhler, 2014) no siempre la revolución educativa fue consecuencia de la revolución industrial y de la revolución democrática - como pensaba Parson (1971) — , sino que por el contrario, en algunas ocasiones, aquella precedió a las últimas, como lo demuestra el caso de Prusia. De esta manera, el análisis del proceso de "educacionalización del mundo" es, en buena medida, la base para la comprensión de los procesos de modernización, es decir, de la consolidación del capitalismo y de la democracia como los sistemas - económico y político - predominantes en el mundo contemporáneo.

Además de introducir una nueva problemática, el libro de Tröhler pone a funcionar un novedoso enfoque metodológico que Thomas Popkewitz (en el prefacio del libro) destaca como una contribución para los estudios actuales, pues el autor "hace una minuciosa lectura por los ámbitos de la filosofía, la historia, las teorías lingüísticas, de la cultura y la sociología" de tal forma que consigue "evitar el provincialismo intelectual [...] en que los estudiosos se interpretan bien en sus «círculos» (lo que en las tesis se llama «revisión bibliográfica»), pero ignoran las interconexiones con otros estudios [...]" (Popkewitz, 2013, p. 9). Esa aportación de Tröhler encuentra su principal realización en la fórmula "lenguajes de la educación" que deriva de su lectura de Saussure, específicamente, de la distinción que hace el lingüista entre langue (lengua) y parole (habla). Con los lenguajes de la educación Tröhler quiere centrar su atención en el cómo y no en el qué, pues no se trata de dar cuenta de las "ideas, conceptos o razonamientos singulares representados por filósofos, políticos o educadores", sino de "los modos o modalidades distinguibles de pensar, hablar o escribir sobre la educación" (2013, p. 23). Así, busca develar las raíces ocultas religiosas, particularmente protestantes, de nuestra actual forma de pensar la educación, y de manera más general, comprender el proceso de educacionalización del mundo, es decir, entender

\begin{abstract}
por qué las personas comparten convicciones al identificar determinadas circunstancias sociales como cuestiones educativas o quizás en un sentido más empírico, por qué empiezan a pensar que ciertas circunstancias deben ser identificadas como cuestiones educativas que incluyen una diversidad de patrones, y cómo estos patrones de pedagogización evolucionaron en el tiempo hasta la actualidad (Tröhler, 2013, p. 23).
\end{abstract}

En sentido general, para Saussure la lengua es como el "almacén pasivo" del sistema de lenguaje, mientras que el habla es la "fuerza activa"; la lengua es un sistema de signos, mientras que el habla es la manifestación particular de ese sistema en un acto de habla, lo cual significa que la primera precede a la segunda; son dos partes que mantienen una interdependencia y, por tanto, la lingüística debe estudiarlos en su especificidad para poder dar cuenta de un sistema lingüístico. Siguiendo esta idea, Tröhler pretende identificar los 
lenguajes (langues) educativos a partir del estudio de las hablas (paroles) de tal forma que se puedan superar los estudios que se centran en el análisis de ciertas lenguas (francés, inglés, alemán). En palabras del autor, con este enfoque, "poco conocido",

la ventaja de observar los lenguajes en lugar de los argumentos -0 mejor, las langues en lugar de las paroles - es como mínimo doble: en primer lugar, los lenguajes son principalmente transnacionales y, por tanto, trascienden el alcance nacional dominante de la investigación educativa. En segundo lugar, dado que solo hay un número limitado de lenguajes, el análisis de estas actitudes normativas fundamentales ofrece el mapeado de las fuentes actuales históricas: las propuestas, los argumentos, los sistemas y los conceptos (Tröhler, 2013, p. 39).

Los dos lenguajes identificados por Tröhler son el protestantismo (en sus distintas versiones) y el republicanismo clásico: el llamado "reflejo educativo" aparecería como respuesta del protestantismo a la tensión generada entre el auge del capitalismo comercial en el siglo XVIII y la oposición a este desarrollada desde el punto de vista del republicanismo clásico. Para los representantes de esta perspectiva, los comerciantes (y sus defensores) no podían contribuir al bien común, pues se regían por el interés particular, por la pasión del dinero, de tal manera que representaban un peligro para la república. Ante la figura del comerciante y del empresario, los republicanos abogaban por un ideal patriótico representado en una persona cabalmente moral, capaz de cumplir con todas sus obligaciones públicas, es decir, un ciudadano orientado al bien común, virtuoso y honesto, figura que personificaba el propietario de tierras y el agricultor.

Según los análisis de Tröhler $(2013,2104)$, las particularidades de esta disputa en Suiza fueron las que llevaron al giro educativo que, finalmente, inició el proceso de educacionalización del mundo. Por una parte, señala Tröhler, estaba el crecimiento sistemático de la población, de la industria de hilados y tejidos y del comercio en Zúrich que permitieron una considerable acumulación de riqueza y su posterior consolidación como exportadora de capital (préstamos), no solo a compañías comerciales sino a las potencias europeas. Por otra parte, se crea un fuerte movimiento anticapitalista que aboga por la restauración del espíritu patriota del ciudadano virtuoso. Esta tendencia, al vincularse con el espíritu protestante de Calvino y Zuinglio, llevó a la idea de que la alternativa al avance del comercio era el fortalecimiento interior, es decir, el fortalecimiento del alma: "Este objetivo protestante en el alma de la persona se convirtió en el punto de partida de la educacionalización del mundo, en la medida que el alma pasó a ser el objeto esencial de la educación" (Tröhler, 2014, p. 23). Debido a las diferencias entre el protestantismo alemán (luterano) y el protestantismo reformado suizo (calvinista y zuinglista), se configuraron dos "ideologías educativas" distintas: la Bildung - "contemplativa y políticamente indiferente" (Tröhler, 2014, p. 23) - y el programa educativo suizo fundamentado en intelectuales como Isaak Iselin, Johann Jacob Bodmer, J.J. Rousseau y J.H. Pestalozzi, centrado en la idea de formar un ciudadano activo.

Reconociendo la pertinencia y potencialidad del concepto de educacionalización para la Historia de la Educación y para la Pedagogía, el presente artículo se propone describir la procedencia y emergencia de las matrices normativas modernas que se encuentran en el núcleo del "giro educativo" señalado Tröhler $(2013,2014)$ en la perspectiva de releer tal acontecimiento a la luz de algunas herramientas arqueogenealógicas propuestas por Michel Foucault. Como principal resultado de ese trabajo de relectura podemos afirmar - a diferencia de Tröhler - que la "educacionalización" del mundo no se inició entre los siglos XVIII y XIX, sino que tuvo su emergencia entre los siglos XVI y XVII en el marco de los 
acontecimientos que Foucault denominó como "la era de la gubernamentalidad" (2006) o que Sloterdijk (2012) designó como el "giro ascetológico" moderno. Se trata de un ejercicio histórico-filosófico con el que queremos mostrar las posibilidades que para pensar la educación y la pedagogía tienen los estudios realizados por Foucault, pero a la vez, la importancia que puede tener el análisis de las prácticas pedagógicas ${ }^{3}$ en el estudio de los problemas que plantea la gubernamentalidad y la ascetología general. ${ }^{4}$ Para llevar a cabo tal ejercicio, reemplazaremos, entonces, la fórmula "lenguajes de la educación" de Tröhler, por las herramientas foucaultianas "gubernamentalidad" y "práctica" (bajo el término específico de prácticas pedagógicas).

\section{Procedencia y emergencia de técnicas propias de las prácticas pedagógicas modernas}

La inquietud específicamente moderna por la educación se articuló alrededor de preguntas por los ejercicios, los comportamientos, las rutinas y los hábitos que los sujetos requerían para conseguir una determinada forma, en particular, para su propio proceso de individualización. El conjunto de técnicas que se pusieron en marcha para la conformación de individuos y la formación de determinadas aptitudes y habilidades no fueron del todo originales, pues ya funcionaban en el interior de los monasterios medievales; fue ese hecho, precisamente, una de las condiciones de posibilidad para la emergencia de las matrices normativas de las prácticas pedagógicas modernas.

La procedencia y emergencia ${ }^{5}$ de dichas matrices puede ser leída a través de la serie individualización-conducción-ejercitación, o mejor aún individuo-conducción-ascesis, que posibilitó el desarrollo del proceso que llamaríamos "educacionalización" del mundo, proceso que, en otros términos, puede entenderse como de constitución de las "sociedades modernas". En la emergencia de las matrices normativas se encuentra una cuestión que no es menor y ella tiene una historia que nos permite asegurar que no nació de una sola vez, pero que tampoco existió siempre, por lo menos no antes de que la "población" se constituyera en objeto de gobierno o conducción (Foucault, 2006). En otras palabras, podemos afirmar que la expansión de las disciplinas y el aumento de la población (en términos de crecimiento demográfico) son asuntos inmanentes y por tanto inseparables: ellos se refieren a un proceso que atravesó la conformación de las sociedades occidentales, un proceso de individualización amplio y extenso que emergió, lentamente, entre los siglos XIV y XVII como correlato de la incorporación de técnicas disciplinarias, derivadas de las formas de vida común religiosas, para el gobierno de amplias masas de población. El preludio de este proceso de "gubernamentalización" (Foucault, 2006) fue el traslado, incorporación y ajuste de dichas técnicas y de un conjunto de ejercicios ascéticos a

\footnotetext{
${ }^{3}$ Sobre el concepto de práctica pedagógica que sirve de sustento para los analisis que aquí ver Marín-Díaz y Noguera-Ramírez (2017a, 2017b).

${ }^{4}$ Este tema ha sido desarrollado en trabajos anteriores, principalmente los siguientes: Noguera $(2015,2017)$; Noguera y Parra (2015); Marín-Díaz (2017); Noguera-Ramírez y Marín-Díaz (2015).

${ }^{5}$ Los conceptos procedencia y emergencia que sustentan este análisis y que orienten las investigaciones de las cuales él surge son usados siguiendo la perspectiva arqueológica y genealógica de M. Foucault. Analizar la procedencia de un deteminado conjunto práctico supone "encontrar bajo el aspecto único de un carácter, o de un concepto, la proliferación de sucesos a través de los cuales (gracias a los que, contra los que) se han formado. [...] es percibir los accidentes, las desviasiones ínfimas —o al contrario los retornos completos-, los errores, los fallos de apreciación, los malos cálculos que han producido aquello que existe y es válido para nosotros; es descubrir que en la raíz de lo que conocemos y de lo que somos no está en absoluto la verdad ni el ser, sino la exterioridad del accidente (Foucault, 1992, p. 13). Por su parte, la emergencia se refiere a la "entrada en escena de las fuerzas; es su irrupción, el movimiento de golpe por el que saltan de las bambalinas al teatro, cada una con el vigor y la juventud que le es propia. [...] la emergencia designa un lugar de enfrentamiento; [...] un no lugar, una pura distancia [...]. Nadie es pues responsable de una emergencia, nadie puede vanagloriarse; esta se produce siempre en el intersticio" (p. 16).
} 
comunidades laicas, con la consecuente definición de "métodos disciplinarios concernientes a la vida cotidiana y a la Pedagogía" (Foucault, 2007a, p. 60).

Hunter (1998) define ese traslado de técnicas como un proceso de laicización del poder pastoral que supuso la entrada de técnicas ascéticas al mundo social, y su posterior expansión y, podríamos agregar, "desespiritualización" — producción de nuevos fines para tales técnicas - bajo la forma de prácticas disciplinarias. En palabras de Sloterdijk (2012), este periodo puede entenderse como una era técnica por excelencia, no tanto por la invención y uso de la imprenta, ni por la trasformación y los descubrimientos derivados de los viajes, la astronomía, la matemática, entre otros, sino, y principalmente, por ser ese el periodo de desarrollo e implementación masiva de diferentes técnicas de producción de lo humano por los propios humanos, es decir, por la implementación masiva de "antropotécnicas".

La amplia difusión y colectivización de las técnicas disciplinarias y las prácticas de ejercitación supuso una de las transformaciones más radicales en la vida humana e hizo de los últimos cinco siglos un periodo marcado por la constitución de diferentes escenarios que permitieran el entrenamiento colectivo e individual. Estado y escuela modernos se configuraron, entonces, como maquinarias adecuadas para la producción de una forma específica de humanidad alcanzando a un amplio número de individualidades somáticas y permitiendo la constitución de subjetividades gobernables orientadas por un "imperativo metanoético" (Sloterdijk, 2012, p. 427) con una doble función: individualizadora productora de individuos (sujetos) - y totalizadora — productora de sociedad-.

La producción de ejercitantes se encuentra en el centro de una de las primeras formas de vida colectivas modernas en la cual los sujetos trabajaban en beneficio propio y en función del Estado y sus instituciones; tal relación fortaleció los procesos de individualización y los de institucionalización de las funciones y relaciones sociales, y supuso el fortalecimiento de técnicas y estrategias disciplinarias orientadas a la producción de individuos y grupos humanos gobernables (Marín-Díaz, 2015). El correlato de este proceso fue un crecimiento demográfico sin precedentes, referido no solo al aumento significativo de singularidades corporales, sino, y principalmente, al de individuos o de individualidades - funciones de sujeto en un cuerpo determinado - que, paradójicamente, a lo largo del último siglo, llevó al cuestionamiento de las instituciones que produjeron esas formas de individualidad y de sociedad modernas. Durante este período, las prácticas de gobierno fueron cuestionadas y la imposibilidad de una regulación completa de la vida individual y colectiva obligó importantes transformaciones en las estrategias de conducción que continuaron vinculadas con las políticas para el gobierno de la vida, llevando, entre otros asuntos, a la discusión sobre el Estado Social y los Derechos humanos (Foucault, 2007b). En otras palabras - y siguiendo a Foucault - la "sociedad disciplinaria", en su desarrollo y extensión, dio lugar a la emergencia de una nueva razón de gobierno, el gobierno liberal en cuyo núcleo la relación individuo-sociedad fue interrogada con lo cual se hizo necesario el ajuste y transformación de las primeras antropotécnicas modernas (las disciplinas) y la aparición de nuevas técnicas de conducción "liberales" como la producción de un "medio" para el control (regulación) de los individuos.

Sobre estas antropotécnicas es preciso señalar que poseen "las características de lo técnico, el dominio de un procedimiento que traerá el resultado buscado mediante pasos discretos, explícitos y controlados" (Sloterdijk, 2012, p. 439). Las disciplinas posibilitaron no solo la producción de seres humanos adecuados y funcionales - masas trabajadoras, estudiantes, militares que alimentaron la maquinaria estatal y las instituciones que se hicieron cada vez más necesarias para conducir las poblaciones - sino, también, una serie de procedimientos educativos empeñados en la fabricación de esos humanos, por medio de una retirada del mundo, ya no al estilo de los antiguos ascetas, sino a través de instituciones de encerramiento y aislamiento como la escuela. En este sentido, es importante señalar que la institucionalización es una de las principales características de estas antropotécnicas modernas 
y quizá la escuela — con sus técnicas de encerramiento, vigilancia, enseñanza y castigo - sea una de sus formas más representativas. Se trata de una de las instituciones cuya operación garantizó la producción de seres humanos, esto es, de sujetos provechosos o, por lo menos no peligrosos para el orden social. Con esta máquina disciplinaria se apostó por la captura total (exhaustiva) de los cuerpos, de los gestos, del tiempo y, en general, de la vida de cada singularidad somática (ni individuo, ni sujeto).

La escuela se organizó alrededor de una práctica que articuló técnicas y estrategias como la observación constante, la ejercitación progresiva y la esquematización normalizadora. La observación constante tenía como propósito garantizar un "estado terminal óptimo" que permitiera "mira hacia el porvenir, hacia el momento en que todo funcione por sí solo y la vigilancia no tenga más que un carácter virtual", esto es, "cuando la disciplina se convierte en un hábito" (Foucault, 2007a, p. 67). La ejercitación progresiva que en una "escala temporal" permita percibir el resultado del "crecimiento y perfeccionamiento de la disciplina" (p. 76) como pieza clave para alcanzar la autonomía e independencia del sujeto. La esquematización normalizante que codifica y crea sistemas de subordinación y clasificación reglados, permite asignar un lugar para cada cosa y cada individuo, incluso para lo inclasificable, lo irreductible a la norma y que, aún así, hace operar el sistema en función de un "trabajo constante de la norma en la anomia" (p. 76). Estos principios disciplinarios encontraron en la escritura una práctica fundamental para codificar, bosquejar y valorar conductas tanto en la institución escolar como en otras instituciones encargadas de producir los sujetos modernos: "la visibilidad del cuerpo y la permanencia de la escritura van a la par y producen [una...] individualización esquemática y centralizada" (p. 69). La escuela y las otras instituciones disciplinarias usaron la escritura como una potente práctica que permitió operar técnicas de vigilancia, ejercitación y normalización para "la modificación exhaustiva de las relaciones entre la singularidad somática, el sujeto y el individuo" (p. 76).

Es clave aclarar que la relación singularidad somática-individuo-función-sujeto es un efecto de las antropotécnicas disciplinarias, pues ellas produjeron un ajuste entre la forma biológica (singularidad somática) y la forma política (individuo), a través de la vinculación a una función sujeto o una individualidad disciplinada adecuada a la organización social: el sujeto moderno. El individuo fue el efecto antropotécnico disciplinar que fijó una funciónsujeto a una singularidad somática: ahora bien, no fue la única o la primera forma de individualización, fue aquella que emergió y se privilegió entre los siglos XV y XVII en lo que podemos reconocer como momento de constitución de las primeras sociedades modernas occidentales. De otra forma, se puede decir que el individuo no preexiste al sujeto ni al uso de las técnicas normalizadoras propias de la racionalidad de gobierno disciplinar (moderna): es un efecto político (subjetivizado) que hizo que la singularidad somática se convirtiera en portadora de una función sujeto. El individuo jurídico es la forma que tomó esta figura histórica en el eje del saber — de la teoría filosófico-política- y tiene su correlato, en el individuo disciplinado (ciudadano) producido en el orden concreto de la vida práctica.

Las sociedades y los individuos en su compleja relación son el producto de una red institucional, pero el despliegue de todo su arsenal antropotécnico disciplinario produjo excedentes inesperados, entre ellos, una sobreproducción biológica de singularidades (cuerpos), asociada a la también sobreproducción subjetiva de individualidades (sujetos). Este fue el escenario para la emergencia, entre otras cosas, de prácticas (y discursos) acerca de la defensa y protección de la individualidad en contra de la intervención Estatal, las cuales adquirieron distintos énfasis y ampliaron el vocabulario y la legislación política y educativa de los dos últimos siglos, en lo que significó la emergencia y consolidación de una razón gubernamental liberal —en términos de Foucault (2007b) — o de "educacionalización del mundo" — en palabras de Tröhler $(2013,2014)$ - 
El análisis de la procedencia y configuración de las matrices normativas de las prácticas pedagógicas ofrece elementos para comprender la productividad de un conjunto de preceptos derivados de la tradición, actualizados en su práctica y apoyados en diferentes instituciones que orientan, limitan, restringen o autorizan las formas como nos conducimos en tanto sujetos (Foucault, 1986); con un estudio como este es posible reconocer principios de funcionamiento y técnicas como el aparejamiento tiempo-progreso, el enclaustramiento y la dirección constante, que orientaron la producción de un entramado de normas y que definieron lo aceptable en la formación y en el comportamiento de los individuos (Foucault, 1986).

Antes de revisar estos principios y técnicas es importante señalar que la operación de las matrices normativas, en tanto juego tenso de normas que orientan la conducta humana, se puede rastrear a través de los códigos y disposiciones explícitas — de las instituciones educativas, judiciales, etc.- así como del conjunto de reglas, principios y preceptos de compartimiento, casi nunca manifiestas, que hacen visibles técnicas y procedimientos de conducción de los sujetos, más allá de las disposiciones institucionales (Foucault, 2009). En otras palabras, se trata de reconocer, por lo menos, dos formas de la norma: una de carácter legal y otra de carácter reglar. La primera referida a formas nominales, aquellas formuladas e institucionalizadas en términos del derecho y la jurisprudencia y que interpelan al individuo en tanto sujeto de derecho; la segunda, derivada de modo de vida práctico monacal que supone hacer de la regla la propia forma de vida: "Una norma que no se refiere a actos y eventos singulares, sino a la existencia toda del individuo, a su forma vivendi" (Agamben, 2013 , p. 44); una regla que es vida y una vida que es regla, una vida "que se vincula tan estrechamente a su forma que se hace inseparable de ella" (p. 7).

En este punto una hipótesis se esboza: la procedencia monacal de las (antropo)técnicas disciplinares dejó en ellas una marca reglar (un efecto técnico difícilmente descriptible) que continúa operando en las prácticas educativas. Así, es posible encontrar en los discursos pedagógicos conceptos - hábito, costumbre, interés, conducta, etc.- que atraviesan las reflexiones sobre la educación, la formación y la enseñanza, señalando una preocupación por la producción de lo humano, asunto que va más allá del problema del derecho y cuyo núcleo es la "idea de la completa maleabilidad de naturaleza humana" cuestión que sirvió de "base para afirmar la omnipotencia de la educación en la conformación de la sociedad humana y [de] fundamento para proclamar la infinita capacidad de perfeccionamiento de la humanidad" (Dewey, [1922] 2014, p. 122).

Retomando la discusión sobre la procedencia y configuración de las matrices normativas de las prácticas pedagógicas y con ellas de la educacionalización del mundo, el ejemplo usado por Foucault (2007a) sobre el proceso de disciplinarización de la juventud estudiantil practicado por la comunidad de los Hermanos de la Vida Común, permite reconocer tres principios técnicos que producidos en las prácticas ascéticas medievales, se trasladaron y ajustaron en comunidades laicas, colectivizándose a través de prácticas pedagógicas cuyo ejercicio no solo estuvo vinculado con la escuela, sino con el amplio entramado de instituciones que ellas contribuyeron a configurar y que se encuentran en el centro de las formas de vida que reconocemos como modernas.

El primer principio es la de aparejamiento tiempo-progreso. Se trata de una técnica ascética que caracteriza la práctica colectiva de un ejercicio individual cuyo propósito es la salvación como efecto de la propia transformación. La división por edades y por niveles, así como los programas de ejercicios progresivos, son principios organizadores de las escuelas fundadas por los Hermanos de la Vida en Común - Deventer, Lieja y Estrasburgo - que vemos operar en las prácticas pedagógicas para garantizar la ejercitación adecuada y el reconocimiento de un aprendizaje que solo podía ocurrir al pasar por etapas obligatorias y necesarias, en tiempos y espacios determinados. Este principio organizador que es evidente en la escuela, también opera en las prácticas no escolares. En los Pensamientos sobre la educación 
Locke ([1690] 2012), por ejemplo, la idea de edades adecuadas para crear hábitos, así como la de una secuencialidad de las acciones se verifican en enunciados que señalan que "la naturaleza puede acostumbrarse a muchas cosas que parecen imposibles, siempre que se procure habituarla desde el comienzo" (p. 37). Ese "comienzo" no solo se refiere a un momento específico en el proceso, un primer momento de la educación que requiere reglas y regulaciones externas, sino a los primeros años como el momento para la formación de hábitos.

Este principio organizador de carácter disciplinar, continúa presente en las prácticas educativas hasta hoy y no fue ajeno a los discursos escolanovistas de finales del siglo XIX y comienzos del siglo XX, aquellos que se propusieron actualizar las prácticas escolares acusadas de "tradicionales". Claparède ([1932] 2007), por ejemplo, señala la importancia que para el método funcional tiene "percibir los procesos en función de la conducta que deban determinar" (p. 69), pues en la práctica, el valor de un proceso lo determina el fin que se quiera alcanzar, y ese fin es alcanzable siempre que se garantice la educación, o sea "una adaptación progresiva de los procesos mentales a ciertas acciones determinadas por ciertos deseos" (p. 201).

Esa idea de gradualidad y progresión que caracterizan la ejercitación (educación), en tanto formas de moldeamiento de la vida, a través de actividades repetidas y reguladas, supone cierta repetición y regularidad en las que se evidencia, por un lado, la plasticidad como una característica humana fundamental y por otro, la retroactividad como un efecto determinante de todo ejercicio practicado por el individuo (Sloterdijk, 2012). Estas características aparecen en diferentes reflexiones pedagógicas vinculadas a un conjunto de nociones que contribuyeron a la producción del horizonte conceptual de la pedagogía: hábito, conducta, plasticidad, ejercicio, vigilancia, disciplina, proceso, etc. En Locke ([1690] 2012), por ejemplo, se encuentran enunciados que señalan que "conforme aumentan los años, es preciso dejarles más libertad y abandonarles en muchas cosas a su conducta propia, puesto que no pueden estar siempre sometidos a una vigilancia excepto la que hayamos puesto en su ejercicio mediante los buenos principios y hábitos establecidos" (p. 44); y más adelante reafirma que "La principal cosa que se debe atender en la educación de los niños es a los hábitos que se les haga contraer en un principio" (p. 53), "la gran falta que he observado en el modo como las generaciones educan a sus hijos, es que [...] no se sabe formar su espíritu en la disciplina, habituarlos a plegarse ante la razón, en la edad en que son más tiernos, más flexibles" (p. 66).

En las reflexiones de Claparède y Dewey el principio de plasticidad vinculado a la idea de gradualidad y progresión fue fundamental. En el primer caso, en un famoso texto sobre Rousseau y la concepción funcional de la infancia, este pedagogo argumenta que Fiske, en 1874, ya había señalado el importante papel que la infancia desempeña en la evolución humana y esto, principalmente, porque se trataba de un periodo de plasticidad "eminentemente favorable para el desarrollo de las facultades físicas y mentales" ([1932] 2007, p. 102). En otro sentido, pero destacando también esta característica asociada al tema de la formación de hábitos, Dewey argumenta que lo "más valioso de la plasticidad está en su capacidad de formar hábitos de juicio independiente y de iniciación inventiva, ya que se requiere una docilidad más completa e intensa para formar hábitos flexibles y fácilmente reajustables, que para adquirir los que copian estrictamente las costumbres de los otros" ([1922] 2014, p. 113).

El segundo principio técnico que opera en las matrices normativas es el enclaustramiento. Este supone reconocer que toda práctica ascética, así como un buen número de prácticas pedagógicas "debe cumplirse en un espacio clausurado, un medio cerrado sobre sí mismo y con un mínimo de relaciones con el mundo externo" (Foucault, 2007a, p. 88). La exigencia de un lugar privilegiado en el proceso de disciplinarización de la juventud estudiantil significó la constitución de los colegios y la ruptura de los lazos que durante el Medioevo las universidades mantenían con el entorno y las clases populares. La técnica de enclaustramiento, de encerramiento o si se quiere la técnica "celular" (Foucault, 2006) es característica de la principales antropotécnicas disciplinarias y, tal vez, su principal expresión se encuentra en la 
forma-escuela como vimos antes, pero ella no fue la única. Al respecto, es preciso señalar que aunque en Comenio (1592-1670) se pueden reconocer los principios prácticos que rodearon aparición del typographeum vivum que fue y es hoy la escuela: "«un taller tipográfico viviente» [...] una maquinaria de aprendizaje moderno al por mayor" (Sloterdijk, 2012, p. 401), su pampadeia no se basa solo en este principio de encierro; la extensión de la forma escuela más allá de sus fronteras llevó, por una parte, al encierro en espacios más amplios (la ciudad como espacio educativo, por ejemplo) y por otra parte, al encierro dentro de sí (la identidad y el yo sobre los cuales se sustentan las formas de vida contemporáneas).

La dirección constante es el tercero y último principio técnico que se puede señalar como característico de las matrices normativas que emergieron en la modernidad. En este caso se trata de reconocer que, así como las prácticas ascéticas requieren de la orientación de otro para que el individuo lleve a cabo los ejercicios que le permitan operar una transformación de sí mismo, las prácticas pedagógicas no pueden ocurrir sin la conducción de ese otro: un guía, un preceptor o un maestro que asuma la responsabilidad de dicha conducción. La guía constante también fue una de las novedades que introdujeron los Hermanos de la Vida Común en los colegios que fundaron y que transformó los modos como era practicada la enseñanza en la Universidad medieval. La incorporación del profesor que sigue y orienta el proceso del individuo en el paso de una etapa a otra, o en la carrera escolar de otro es además la incorporación de un testigo del cumplimento de todos los niveles y con ello del progreso alcanzado por aquel ejercitante que es el escolar.

La figura del maestro, del profesor o del docente (del adulto, en términos generales) refiere a posiciones de sujeto diferentes en la práctica pedagógica. Cada una de ellas remite no solo a unas funciones sino a unos modos de relación específica tanto con la institución como el estudiante e implica reconocer que no es una práctica personal o individual, sino una práctica derivada de una posición ocupada y que, aunque se ajusta en condiciones y formas de institucionalidad específicas, no es independiente de las matrices normativas y las formas de saber de un momento y sociedad determinada. Así, la formas de ser sujeto adulto (profesor, maestro, docente) de una práctica, aunque se actualizan con la presencia y algunos modos concretos de actuar de los individuos, no dejan de estar regidas y definidas por las condiciones que la posibilitan y que se transforman con la participación de los individuos que se subjetivan con ella, independiente de su voluntad de transformación.

En términos generales, y a manera de conclusión se pueden señalar tres asuntos que resultan de esta mirada histórica a las prácticas pedagógicas, a través del lente que ofrece la serie individualización-conducción-ejercitación. Primero que la emergencia de las matrices normativas que definieron las prácticas pedagógicas modernas y acompañaron el giro educativo son perceptibles en por lo menos dos dimensiones: por un lado, la institución que se refiere a las normas escolares, sus tiempos y espacios claramente establecidos; por el otro lado, la vida práctica como lugar de procedencia y emergencia de un buen número de reglas incorporadas, de hábitos, principios y preceptos de comportamiento que definen tanto lo aceptable o no para el individuo y el grupo social como lo que se considera propiamente humano. Segundo, que la matriz normativa de la cual emergieron las prácticas pedagógicas modernas es histórica y que su modificación ocurre como parte de la actualización del dispositivo al que se encuentra vinculada (disciplinar, liberal, etc.). Finalmente, y tercera, que las formas de conducción modernas, articuladas a las matrices normativas que emergieron entre los siglos XVI y XVII, se caracterizan por formas de normalización a través de las cuales se constituyen individualidades y formaciones sociales que definen los modos como somos sujetos modernos, así como las maneras como nos conducimos y condujimos a otros hace casi cuatro siglos. 


\section{El proceso de educacionalización del mundo: gobierno o educación de todos y cada uno}

Hemos realizado una aproximación a la procedencia y emergencia de las técnicas que están en el centro de las prácticas pedagógicas modernos, ahora realizaremos una relectura de ese proceso, ya no desde la perspectiva de las técnicas involucradas, sino desde la estrategia general que, siguiendo a Foucault, llamaríamos "gubernamentalización" del Estado y de la sociedad. Para ello es necesario tener en cuenta la crisis de gobierno del siglo XVI. Sobre este acontecimiento Foucault (2006) señala que, de manera simultánea, acerca de muchas cuestiones diferentes y con distintos acentos, aparecen durante el siglo XVI en Europa, crisis que van desde el orden del gobierno de sí mismo, del gobierno de las almas y de las conductas, hasta el gobierno de los niños. Emerge allí una gran problemática pedagógica, tal como aparece y se desarrolla en el siglo XVI con la Reforma y, posteriormente, con la Contrarreforma.

Esa crisis de gobierno señala el inicio de un proceso de gubernamentalización del Estado y la aparición de un nuevo tipo de poder, el gobierno, cuya procedencia se encuentra en el poder pastoral. Durante la Edad Media el soberano reinaba, pero no gobernaba, pues el gobierno, es decir, la conducción o dirección de los otros, era un asunto del pastor (de la Iglesia y sus ministros). Pero a partir del siglo XVI, con la denominada crisis de gobierno, se da inicio a un proceso de laicización del poder pastoral que llevará a la aparición del gobierno como una nueva forma de poder y con ella, de una preocupación por la conducción de los otros (y de sí mismo), asuntos que se perciben en los movimientos de reforma protestante, en la constitución de la Razón de Estado, en las prácticas de "policía" (disciplinarias) implementadas para el control de las poblaciones y en las acciones educativas dirigidas a obreros, indígenas, pobres y nobles en Europa y sus territorios de influencia (Foucault, 2006; Marín-Díaz, 2015).

El inicio del proceso de gubernamentalización puede ser entendido, a su vez, como el proceso de creación de una sociedad disciplinaria o como el correlato de la emergencia e instalación de las matrices disciplinarias, esto si se tiene en cuenta el papel central que las "disciplinas" jugaron en el control y gestión de las poblaciones a partir del siglo XVI. Ahora bien, el instrumento clave para la expansión de las disciplinas (y del gobierno como nueva forma de poder) entre la población fueron las prácticas pedagógicas (entre ellas la escolarización). Por sus propósitos y características, las prácticas pedagógicas son prácticas de gobierno, es decir, prácticas de conducción de la conducta (de otros y de sí mismo) y es posible reconocer su aparición entre los siglos XVI y XVII a propósito de dos nuevos conceptos: institutio y eruditio (Noguera, 2011, 2012) valga decir, la idea sobre la necesidad de "instituir" (educar) los niños de Montaigne y Erasmo, y la idea sobre necesidad de "enseñar" (instruir en) todo a todos de Comenio. Se trata de dos ideas novedosas para la época que, sin embargo, tienen su procedencia en prácticas antiguas (paideia griega y cristiana): de la primera de ellas procede el concepto moderno de educación, de la segunda, el de enseñanza.

Siguiendo esta ruta de análisis, el proceso de gubernamentalización o la creación de una sociedad disciplinaria marcaría el inicio del "giro educativo" (modernidad) que puede ser leído, a su vez, como el proceso de educacionalización del mundo. A diferencia de Tröhler, no se trata de lenguajes sino de discursos (en el sentido de Foucault): el giro educativo se entiende como la aparición de un discurso pedagógico que se expresa, inicialmente, en dos conceptos (institutio y eruditio) (Noguera, 2011, 2012) que, aunque formulados en latín, son independientes de la lengua y de la nacionalidad de sus "autores". Posteriormente, hacia el siglo XVII, las ideas de Montaigne y Erasmo sobre la "institución" de los niños nobles serán sistematizadas por Locke en el concepto de educación que desarrolla en su libro Some Thoughts Concerning Education (1693) y a mediados del siglo XVIII, Rousseau en su Émile ou de l'éducation (1762) lo redefinirá bajo la órbita de una nueva forma de gobierno (liberal) 
en donde la capacidad de agencia del sujeto y el control del medio (y ya no la disciplina) ocupan el lugar principal.

En todos estos autores existe la idea de que, dada su naturaleza, el hombre debe alcanzar la virtud y para ello requiere de la educación (institución). Montaigne, tal vez el primero en utilizar la expresión "educación" en una lengua vernácula, opone ésta a la "pedantería" o falsa "erudición" que promueven los maestros de escuela de su época. La institución (educación) debe orientarse hacia la virtud y la sabiduría en contra de la erudición, de tal forma que una cabeza bien formada es preferible a una bien llena. Erasmo considera que la "institución" es más eficaz que la naturaleza, es decir, que, dado que la naturaleza no dotó al hombre de los instrumentos adecuados para vivir, necesita de la educación a través de la cual puede aprender "todas las disciplinas": "Cuanto menos apto es cada animal para las disciplinas, mejor dotado está de congénita destreza" (Erasmo, 1956, p. 923); o, en otras palabras, "Eficaz res est natura, sed hanc uincit efficacic or institutio [eficaz es la naturaleza, pero la supera en eficacia la educación institución]" (Erasmo, 1529, p. 8). En el siglo siguiente, Locke (1986, p. 131), en sus pensamientos sobre la educación, considera que la labor del preceptor es "la de moldear la conducta y formar el espíritu, establecer en su discípulo los buenos hábitos, los principios de la virtud y de la sabiduría" antes que hacer del joven gentleman un perfecto erudito; por esa razón dejó el tema de la "instrucción" en el último lugar de su libro: "La instrucción es necesaria pero no debe colocarse sino en segundo lugar, como un medio de adquirir cualidades más altas" (p. 208). Varias décadas después, Rousseau, partiendo del trabajo de Locke, avanza en una nueva dirección sobre la base del principio liberal de gobierno: gobernar menos para gobernar, principio que traducido a la educación se explica de la siguiente manera: "En las educaciones más esmeradas, el maestro manda y cree que dirigir: en realidad es el niño el que dirige [...] Tomad un camino opuesto con vuestro alumno; que siempre crea él ser el maestro, y que siempre lo seáis vos. No hay sometimiento tan perfecto como el que conserva la apariencia de la libertad; de este modo se cautiva la voluntad misma [...] Indudablemente no debe hacer más que lo quiere, pero sólo debe querer lo que vos queréis que haga" (Rousseau, 1990, p. 181-182).

Por su parte, la erudición se coloca en el centro de las preocupaciones de Comenio en la medida en que el hombre, para alcanzar su condición de criatura hecha a imagen y semejanza divina, debe convertirse en un ser racional, lo que significa utilizar la razón para conocer las cosas del mundo que el creador ha dispuesto para su disfrute. Si bien es evidente una connotación religiosa en esta idea, también resulta evidente que se trata de un argumento "antropológico", es decir, que la necesidad de "erudición" (o sea, la necesidad de conocer todas las cosas del mundo) y con ella, la necesidad de la enseñanza, forma parte de la naturaleza humana: "Quede, pues sentado, que a todos los que nacieron hombres les es precisa la enseñanza, porque es necesario que sean hombres, no bestias feroces, no brutos, no troncos inertes" (Comenio, 1994, p. 23). A diferencia de Montaigne, Erasmo y Locke preocupados por la educación de los nobles - la necesidad de erudición y de enseñanza es para todos, sin exclusión, pero no por un asunto democrático, sino, como hemos visto, por una razón antropológica. A comienzos del siglo XIX, F. Herbart, con su idea de Pedagogía General, intentó vincular educación y enseñanza en su fórmula "la enseñanza educativa" que significa que, para ser tal, la enseñanza debe educar (no solo instruir) y que no pude existir verdadera educación sin instrucción.

\section{¿Fin de la "educacionalización"?}

La educacionalización del mundo comenzó, entonces, en el siglo XVI, pero tuvo su mayor impulso con Comenio y su campaña de una "educación" universal — pampaedeia-, proyecto inédito en la historia de la humanidad que constituyó el verdadero "giro educativo", 
un "giro hacia algo más grande"6: la emendatio mundi, la mejora del mundo que significa "mejora de la humanidad en masse" (Sloterdijk, 2012, p. 42). Si hasta entonces el imperativo metanoético (de conversión, de transformación a partir de una vida ejercitante) era asunto de unas élites aristocráticas y monásticas, el proyecto de Comenio consistió en la extensión de las ascesis, de la ejercitación, a través de la disciplina y el estudio, a toda la humanidad, sin excepción. Tal proyecto es calificado por Sloterdijk como "la idea más potente, por sus efectos, de los últimos quinientos años" (2012, p. 441). En su Via Lucis, Comenio consideraba al mundo como la "casa disciplinar", "Pues el mundo está integrado, en su totalidad, por un conjunto de docentes, de discentes y de disciplinas. Pues todo lo que hay en el mundo o enseña o aprende o hace, alternativamente, las dos cosas" (citado por Sloterdijk, 2012, p. 446). ¿No es, acaso, esta idea la "educacionalización del mundo"?

Valiéndonos de Foucault y Sloterdijk, hemos leído la aparición de la educación moderna (en su dimensión conceptual y práctica) como el inicio de un proceso de educacionalización del mundo que ha llevado, no solo a que la escuela sea hoy una institución globalmente aceptada y valorada, sino que la educación de todos se haya constituido, durante el siglo XX, como una cuestión primordial en el orden político y económico mundial. Desde esta perspectiva resulta evidente que la llamada modernidad se corresponde con ese proceso de educacionalización (o gubernamentalización), lo que significa que es necesario reinterpretar el lugar de la escuela en los discursos historiográficos y sociológicos que la han considerado como un efecto de la modernización. Habría que decir, por el contrario, que ha sido la educacionalización del mundo lo que ha significado su "modernización". Pero más allá de esta necesaria revisión, lo que está en juego es el análisis del sentido de un mundo educacionalizado, o dicho en términos foucaultianos, el análisis de las actuales formas de gobierno de todos y de cada uno, pues tal educacionalización implicó la instauración (a lo largo de cuatro siglos) de formas disciplinares, liberales y neoliberales de gobierno de los individuos y de las poblaciones.

Fenómenos como el "narcicismo" contemporáneo, descrito por Lipovetsky en la Era del Vacío (2000), o la licuefacción de la modernidad, señalada por Bauman (2004), o la sociedad del riesgo, estudiada por Beck (2002) no pueden entenderse sin tener en cuenta el largo proceso de educacionalización del mundo iniciado con los humanistas del Renacimiento y expandido con los Estados-nación desde el siglo XIX. Evidentemente, se trata de un fenómeno de larga duración que comienza a pensarse hoy con estudios como los que hemos analizado en este texto. Sin embargo, podríamos haber traspasado el punto máximo de la educacionalización; tal vez no sea este el momento de mayor auge; quizás estemos en el borde de un nuevo giro, ya no el "giro educativo" sino, quizás, el "giro del aprendizaje". Si atendemos a lo que algunos colegas han venido anunciando en la última década con expresiones como el "dispositivo de aprendizaje" contemporáneo (Simons; Masschelein, 2013), la "desaparición de la enseñanza y del maestro" (Biesta, 2016), "un "mundo sin adultos" (Narodowski, 2016), o en términos de un premio nobel de economía, el nacimiento de "la sociedad del aprendizaje" (Stiglitz; Greenwald, 2014) o de un sociólogo "la corrosión del carácter" (Sennett, 2010), tal vez ante estas nuevas evidencias que estos autores muestran y analizan, tengamos que aceptar que estamos abandonando el mundo educacionalizado y estamos entrando en el mundo del aprendizaje que sería el mundo de la "pos-educación", pues a partir de los anteriores estudios, resulta claro que no es lo mismo educación que aprendizaje.

El mundo educacionalizado era un mundo con adultos (padres, maestros, autoridad) y un lugar de ejercitación guiada hacia el desarrollo de determinadas formas de ser y hacer fundamentadas en tradiciones; por el contrario, el mundo del aprendizaje, la sociedad del

\footnotetext{
${ }^{6}$ En su texto Prodromus pansophiae Comenio afirma que "ha llegado realmente la hora tanto de esperar como de intentar algo más grande" que es, justamente, la mejora de la humanidad a través de la "educación universal". (Citado por Sloterdijk, 2012, p. 401).
} 
aprendizaje, el mundo "aprendificado" (Biesta, 2016), "la sociedad del cansancio (Han, 2016) es un mundo de individuos emprendedores, empresarios de sí mismos, pero también, de angustiados y deprimidos (Sennett, 2010), autoexplotados e hiperactivos (Han, 2016); individuos que no son más ni infantes ni adultos (Postman, 1999; Narodowski, 1999, 2016) y en donde "nadie educa a nadie" (Rocha, 2017), pero todos debemos aprender permanentemente, a lo largo de toda la vida.

\section{Referencias}

Agamben, G. (2013). Altísima pobreza. Reglas monástica y formas de vida. Buenos Aires: Adriana Hidalgo.

Bauman, Z. (2004). La modernidad líquida. Buenos Aires: Fondo de Cultura Económica.

Beck, U. (2002). La sociedad del riesgo global. Siglo XXI de España Editores.

Biesta, G. (2016). Devolver la enseñanza a la educación. Una respuesta a la desaparición del maestro, en, Pedagogía y Saberes (44), pp. 119-129. https://doi.org/10.17227/01212494.44pys119.129

Claparède, E. ([1932] 2007). La educación funcional. Madrid: Biblioteca Nueva.

Comenius, J. ([1657] 1907). The Great Didactic. London: Adam and Charles Black. [Translated by: M. W. Keatinge]

Depaepe, M; et. al. (2008). About Pedagogization: From the Perspective of the History of Education, in, Smeyers, P.; Depaepe, M. (Editors). Educational Research: the Educationalization of the Social Problems, Vol. 3, Springer.

Dewey, J. (1922). Human Nature and Conduct. An Introduction to Social Psychology. New York: Henry Holt and Company.

Elias, N. ([1939] 1987). La sociedad de los individuos. Barcelona: Península.

Erasmo de Rotterdam. (1956b). "De cómo los niños precozmente y desde su nacimiento deben ser iniciados en la virtud y en las buenas letras". En: Obras escogidas. Madrid: Aguilar, pp. 919-962.

Erasmo. (s/f) [1529]. De pueris (Dos meninos). São Paulo: Escala.

Foucault, M. (1986). Historia de la sexualidad 2-El uso de los placeres. México: Siglo XXI.

Foucault, M. (1992). Nietzsche, la genealogía, la historia. En Foucault, M. Microfísica del poder. Traducción de Julia Varela y Fernando Álvarez-Uria. Madrid: La piqueta, p. 7-30.

Foucault, M. (2006). Seguridad, territorio, población. Curso en el Collège de France (19771978). Traducción de Pons Horacio. Buenos Aires: Fondo de Cultura Económica.

Foucault, M. (2007a). El poder psiquiátrico. Curso en el Collège de France (19731974).Trad. Horacio Pons. Buenos Aires: Fondo de Cultura Económica. 
Foucault, M. (2007b). Nacimiento de la biopolítica. Curso en el Collège de France (19781979).Trad. Horacio Pons. Buenos Aires: Fondo de Cultura Económica.

Foucault, M. (2009). El gobierno de sí y de los otros: Curso en el Collège de France 19821983. Buenos Aires: Fondo de Cultura Económica.

Foucault, M. (2010). ¿Qué es un autor? Trad. Horacio Pons. En Obras esenciales, Barcelona: Paidós, pp. 291-317.

Han, B. (2016). La sociedad del cansancio. Barcelona: Herder.

Hunter, I. (1998). Repensar la escuela. Subjetividad, burocracia y crítica. Barcelona: Pomares- Corredor.

Lipovetsky, G. (2000). La era del vacío. Ensayos sobre el individualismo contemporáeno. Barcelona: Editorial Anagrama.

Locke, J. ([1690] 2012). Pensamientos sobre la educación. Madrid: Akal.

Marín-Díaz. D. (2015). Autoajuda e educação. Genealogia de uma antropotécnica. Belo Horizonte: Autêntica.

Marín-Díaz, D. (2017). The key is the individual: Practices of the self, self-help and learning. Educational Philoshopy and Theory, 49:7, 710-719.

Marín-Díaz, D.; Noguera-Ramírez, C. (2017a). Saberes, normas y sujetos: cuestiones sobre la práctica pedagógica. DOI: https//doi.org/10.1590/0104-4060.53866. Educar em Revista. ISSN 0104-4060; e-ISSN 1984-0411. v.33. n.66. p. 37-56. https://doi.org/10.1590/0104-4060.53866

Marín-Díaz, D.; Noguera-Ramírez, C. (2017b). En defensa de la experiencia escolar. Fortalecer las fronteras de la escuela. DOI: https//doi.org/10.20396/etd.v19i4.8648826. Educação Tematica Digital- ETD. ISSN 1676-2592. v.19. n.4. p. 607-621. https://doi.org/10.20396/etd.v19i4.8648826

Masschelein, J. y Simons, M. (2013). Se nos hace creer que se trata de nuestra libertad: notas sobre la ironía del dispositivo de aprendizaje. Pedagogía y Saberes, 39, 93-102. https://revistas.pedagogica.edu.co/index.php/PYS/article/view/2142

Narodowski, M. (1999). Después de clase. Desencantos y desafíos de la escuela actual. Buenos Aires: Novedades Educativas.

Narodowski, M. (2016). Un mundo sin adultos. Familia, escuela y medios frente a la desaparición de la autoridad de los mayores. Buenos Aires: Debate.

Noguera, C. (2010). La constitución de las Culturas Pedagógicas Modernas: una aproximación conceptual, en Pedagogía y Saberes 33, pp. 9-25.

Noguera, C. (2011). Pedagogia e governamentalidade ou Da Modernidade como uma sociedade educativa. Belo Horizonte: Autêntica. 
Noguera, C. (2012). El gobierno pedagógico. Del arte de educar a las tradiciones pedagógicas. Bogotá: Siglo del Hombre.

Noguera, C. (2017). The pedagogical effect: On Foucault and Sloterdijk, Educational Philoshopy and Theory, 49:7, 720-733

Noguera-Ramírez, C. y Marín-Díaz, D. (2015). Training the human anima: Biopolitics and Anthropotechnics. Sisyphus Journal of Education, 3:3, 110-131.

Noguera, C. y Parra, G. (2015). Pedagogización de la sociedad y crisis de la educación. Elementos para una crítica de la(s) crítica(s), Pedagogía y Saberes 43, p. 69-78. https://doi.org/10.17227/01212494.43pys69.78

Popkewitz, T. (2013). Prefacio. En Tröhler, D. (2013). Los lenguajes de la educación. Los legados protestantes en la pedagogización del mundo, las identidades nacionales y las aspiraciones globales. Barcelona: Octaedro, p. 9-18.

Postman, N. (1999). O desaparecimento da infância. Rio de Janeiro: Graphia.

Rocha, R. (2017). Quando ninguém educa. Questionando Paulo Freire. São Paulo: Contexto.

Rousseau, J. (1990). Emilio o de la educación. Madrid: Alianza.

Sennett, R. (2010). La corrosión del carácter. Barcelona: Anagrama.

Simons, M. y Masschelein, J. (2008). It makes us believe that is about our freedom: notes on irony of the learning apparatus. En Educational research: the educationalization of social problems (pp. 191-204). Springer.

Simons, M. y Masschelein, J. (2013). Se nos hace creer que se trata de nuestra libertad: notas sobre la ironía del dispositivo de aprendizaje. En Pedagogía y Saberes 38, p. 93-102. https://doi.org/10.17227/01212494.38pys93.102

Sloterdijk, P. (2012). Haz de cambiar tu vida. Valencia: Pre-textos.

Smeyers, P. y Depaepe, M. (2008). Educational research: The educationalization of social problems. Springer.

Stiglitz, J. y Greenwald, B. (2014). La creación de una sociedad del aprendizaje. Bogotá: Planeta.

Tröhler, D. (2011). Languages of Education. Protestant Legacies, National Identities, and Global Aspirations. New York: Routledge.

Tröhler, D. (2013). Los lenguajes de la educación. Los legados protestantes en la pedagogización del mundo, las identidades nacionales y las aspiraciones globales. Barcelona: Octaedro.

Tröhler, D. (2013). Pestalozzi and the educationalization of the world. New York: Palgrave Macmillan US.

Tröhler, D. (2014). Pestalozzi y la educacionalización del mundo. Barcelona: Octaedro. 